\title{
Investigation of Inverse Analysis and Neural Network Approaches for Identifying Distributed Load using Distributed Strains*
}

\author{
Daichi WadA, ${ }^{1 \dagger}$ Yohei Sugimoto, ${ }^{2)}$ Hideaki Murayama, ${ }^{3)}$ Hirotaka Igawa, ${ }^{1)}$ and Toshiya NAKAmurA ${ }^{1)}$ \\ ${ }^{1)}$ Aeronautical Technology Directorate, Japan Aerospace Exploration Agency, Mitaka, Tokyo 181-0015, Japan \\ ${ }^{2)}$ Research and Development Directorate, Japan Aerospace Exploration Agency, Tsukuba, Ibaraki 305-8505, Japan \\ ${ }^{3)}$ Graduate School of Frontier Sciences, The University of Tokyo, Kashiwa, Chiba 277-8561, Japan
}

\begin{abstract}
We propose and investigate two approaches to identify load distributions on a flat panel by using strain measurement values. One approach is an inverse analysis that utilizes the inverse matrix of the load and strain relationship, and the other is a neural network approach that trains a neural network using strains as input and loads as output. For both approaches, we propose a method using a pressure discretization map to represent the load distributions as a set of discrete pressure values. This method makes load identification applicable to load distributions with arbitrary profiles. In order to examine and verify the performance, we conducted numerical simulations and an experiment. Numerical simulation results verified both approaches; however, identification results using the inverse approach were unstable when the strain measurement error existed. On the other hand, the neural network approach showed high robustness to the strain errors by training neural networks with data including artificial strain errors. Based on the results, we discuss the applicability of the load identification approaches.
\end{abstract}

Key Words: Inverse Analysis, Load Identification, Machine Learning, Neural Network, Structural Monitoring

\section{Introduction}

For the safe and efficient operation of aircraft, it is essential to monitor the usage of aircraft such as aerodynamic loads on wings in flight. Information of aerodynamic loads can be used to improve limit and fatigue load modeling. Information of individual aircraft fatigue is beneficial for comparing operational and design usage, planning of maintenance, modifying operations and understanding structural problems. ${ }^{1,2)}$ When the monitoring data of loads is combined with damage and fatigue databases complied using various structural health monitoring techniques, ${ }^{3,4)}$ appropriate data management will lead to highly efficient analysis and operation of aircraft such as condition-based maintenance, damage prognosis and structural health management. ${ }^{5,6)}$

Directly measuring load is typically difficult due to the complexity of load measurement instrumentation on the wing. Consequently, loads have to be determined based on other measurable structural responses such as strains. This raises an inverse problem. ${ }^{7-10)}$ In the case of aerodynamic and inertia loads, which are continuously distributed, the inverse problem results in an ill-posed governing system of equations in the sense that they do not necessarily satisfy conditions of existence, uniqueness, and stability. Therefore, a special approach is required to obtain an accurate and stable solution.

Schnur and Zabaras ${ }^{11)}$ and Maniatty et al. ${ }^{12,13)}$ investigated a finite element-based method for inverse elastic problems. In order to improve the robustness of the algorithms, they presented a spatial regularization procedure ${ }^{11)}$ and a regulari-

(C) 2019 The Japan Society for Aeronautical and Space Sciences

*Received 16 May 2018; final revision received 7 November 2018; accepted for publication 18 December 2018.

†Corresponding author, wada.daichi@jaxa.jp zation procedure coupled with a statistical approach. ${ }^{8,12-14)}$ Martin et al. discussed a non-iterative algorithm for determining surface tractions using a boundary element method. ${ }^{15)}$ This algorithm has the restriction that both deformations and tractions must be applied simultaneously on at least a part of the surface structure. Shkarayev et al. developed an inverse interpolation method. ${ }^{16)}$ The load distributions were assumed to be a quadratic function of spatial coordinates. Coates and Thamburaj generalized this method and introduced Fourier cosine series terms for the load distribution functions. ${ }^{17)}$ Nakamura et al. proposed a finite element-based method that expressed continuously distributed loads by interpolating nodal pressures. ${ }^{18)}$ Aerodynamical equations were used to improve the load estimation accuracy. As seen in the various approaches mentioned above, the inverse approach requires the assumption of a certain form of functions for distributed aerodynamic loads to obtain stable solutions. This would limit the applicability of identification when the target load distributions are not described using a simple form of functions.

Other load identification approaches demonstrated by Cao et al. ${ }^{19)}$ and Trivailo and $\mathrm{Carn}^{20)}$ were to avoid the inverse calculation by using artificial neural networks to represent the strain and load relationship in a direct manner; that is, using strains as input and loads as output. The direct strain-load relationship was determined by using a learning algorithm, and maneuver and buffet loads were calculated. They mainly focused on the identification of a single or limited number of discrete concentrated loads. Wada and Sugimoto ${ }^{21)}$ also demonstrated an approach using a neural network. Using a number of strains, they identified distributed profiles of loads through numerical simulations. These studies showed the possibility of neural networks representing the strain-load re- 
lationship; however, there has not been sufficient discussion about the effect of the strain error. The robustness of the load identification calculation has to be investigated to demonstrate feasibility.

In order to enhance the applicability of load identification techniques for various designs of aircraft, a method that can assume any possible load distribution is beneficial. In addition, one of the major concerns about load identification is the sensitivity to the strain errors. Therefore, it is beneficial to compare the inverse and direct (neural network) approaches, and gain a comparative perspective in the sense of the robustness to the strain errors.

In this paper, taking an example of a flat panel under distributed loads, we propose and investigate two approaches to identify load distributions using strain measurement values. One approach is an inverse analysis that utilizes the inverse matrix of the load and strain relationship, and the other is a neural network approach that trains the neural network using strains as input and loads as output. For both approaches, we propose a method using a pressure discretization map to represent the load distributions as a set of discrete pressure values. This method makes load identification applicable to arbitrary profiles of load distributions. In order to verify and examine the performance, we conduct numerical simulations and an experiment. We investigate individual methods for the two approaches to improve identification accuracy when strain measurement errors exist, and discuss optimum use of the load identification approaches.

\section{Materials and Methods}

\subsection{Materials}

We conducted load identification for the case of an acrylic rectangular plate under a distributed load. The plate was simply supported at three points and 60 strain gauges were attached. The length, width and thickness were $2.0 \mathrm{~m}, 1.0 \mathrm{~m}$ and $15.0 \mathrm{~mm}$, respectively. We assigned an $x y z$ coordinate, as shown in Fig. 1. The strain gauges were attached on the bottom surface $(-z)$ and loads were applied on the top surface $(+z)$. Considering the future application of effective sensing techniques such as fiber optic strain-sensing, ${ }^{22)}$ we only utilized the strain data along the $x$-axis.

In the following numerical simulation, we built a finite element model of the plate using a general-purpose code, ABAQUS. The element type was a 4-node shell and the shape was square with the length of $10 \mathrm{~mm}$ on a side. The total number of elements and nodes resulted in 20,000 and 20,301, respectively. The elastic modulus and Poisson's ratio were set as $4 \mathrm{GPa}$ and 0.3 , respectively, and the isotropic material property was assumed. The displacement of the $z$-axis was constrained at $(x, y)=(0.19 \mathrm{~m}, 0.05 \mathrm{~m})$ and $(0.19 \mathrm{~m}$, $0.95 \mathrm{~m})$, whereas the displacements of the $x, y$ and $z$ axes were constrained at $(x, y)=(1.69 \mathrm{~m}, 0.50 \mathrm{~m})$.

In the following experiments, we applied distributed loads using $5 \mathrm{~cm} \times 5 \mathrm{~cm}$ aluminum square plates, as shown in Fig. 2. The aluminum weights were set with $1-\mathrm{cm}$ intervals between each.

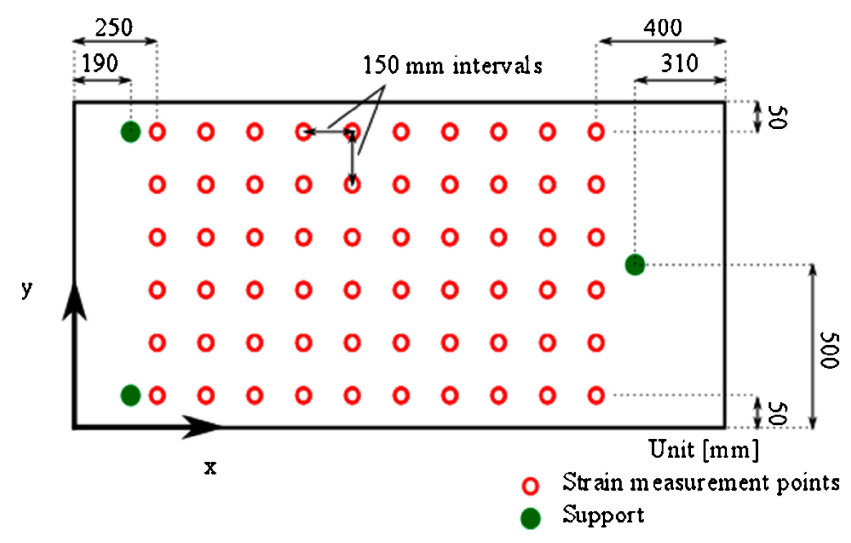

Fig. 1. Schematic of the rectangular plate simply supported at three points.

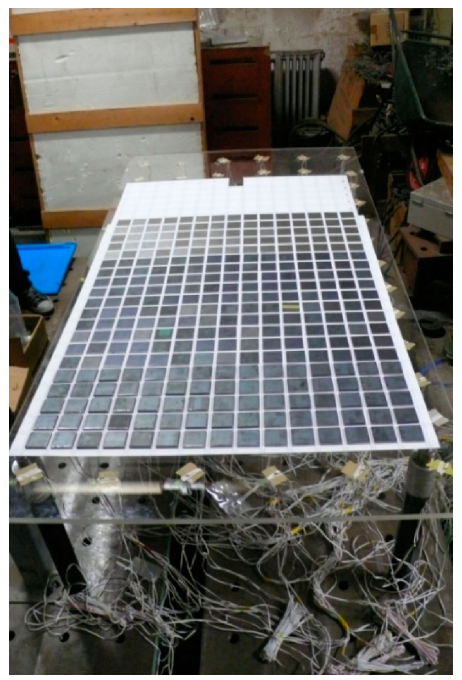

Fig. 2. Picture of the loaded plate. View from $x=0$.

In both the simulation and experiments, we applied loads within the area of $0.19 \mathrm{~m} \leq x \leq 1.69 \mathrm{~m}$ and $0.05 \mathrm{~m} \leq y \leq$ $0.95 \mathrm{~m}$.

\subsection{Methods: Representation of the pressure distribu- tion}

We consider load (pressure) distribution, $p=p(x, y)$, on the $x y$-plane under static conditions. We prepared a map that we call the "pressure discretization map" at $0.19 \mathrm{~m} \leq x \leq 1.69 \mathrm{~m}$ and $0.05 \mathrm{~m} \leq y \leq 0.95 \mathrm{~m}$, as seen in Fig. 3. This is done so that the pressure distribution is represented by discrete loads on each node and the shape function that interpolates the node values. Using a triangular shape, pressure on a triangular element is expressed using the shape function, $u_{i}^{e}(x, y)$, as

$$
p^{e}(x, y)=\sum_{i=1}^{3} u_{i}^{e}(x, y) p_{i}^{e}
$$

where, the superscript notation, $e$, represents the element, and the subscript notation, $i$, represents a node of the element. The shape functions of each node are written as 


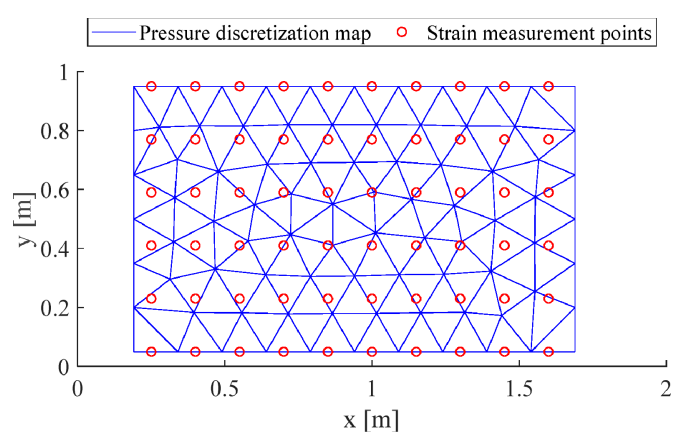

Fig. 3. Pressure discretization map.

The red circles indicate the location of the strain measurement points for reference.

$$
\left\{\begin{array}{l}
u_{1}^{e}(x, y)=a_{1}+b_{1} x+c_{1} y \\
u_{2}^{e}(x, y)=a_{2}+b_{2} x+c_{2} y \\
u_{3}^{e}(x, y)=a_{3}+b_{3} x+c_{3} y
\end{array}\right.
$$

where, $a_{i}, b_{i}$ and $c_{i}$ are constants and written as

$$
\left\{\begin{array}{l}
a_{1}=\frac{1}{2 \Delta}\left(x_{2} y_{3}-x_{3} y_{2}\right) \\
a_{2}=\frac{1}{2 \Delta}\left(x_{3} y_{1}-x_{1} y_{3}\right) \\
a_{3}=\frac{1}{2 \Delta}\left(x_{1} y_{2}-x_{2} y_{1}\right) \\
b_{1}=\frac{1}{2 \Delta}\left(y_{2}-y_{3}\right) \\
b_{2}=\frac{1}{2 \Delta}\left(y_{3}-y_{1}\right) \\
b_{3}=\frac{1}{2 \Delta}\left(y_{1}-y_{2}\right) \\
c_{1}=\frac{1}{2 \Delta}\left(x_{3}-x_{2}\right) \\
c_{2}=\frac{1}{2 \Delta}\left(x_{1}-x_{3}\right) \\
c_{3}=\frac{1}{2 \Delta}\left(x_{2}-x_{1}\right),
\end{array}\right.
$$

where, $\Delta$ is the area of the triangular element. Then, the pressure distribution is expressed by adding the element pressures as

$$
p(x, y)=\sum_{j=1}^{n} U_{j}(x, y) p_{j}
$$

where, $n$ is the number of nodes, $p_{j}$ is the pressure (load) value at each node and $U_{j}$ is calculated by superposition of the shape functions in Eq. (2). In this study, the number of nodes on the pressure discretization map was $n=85$.

Using the above approach, we can represent arbitrary pressure distributions using the set (vector) of discrete values $p_{j}$ $(j=1,2, \cdots, n)$. As examples, Fig. 4(a) shows a pressure distribution when $p_{j}=1$ at a node located approximately

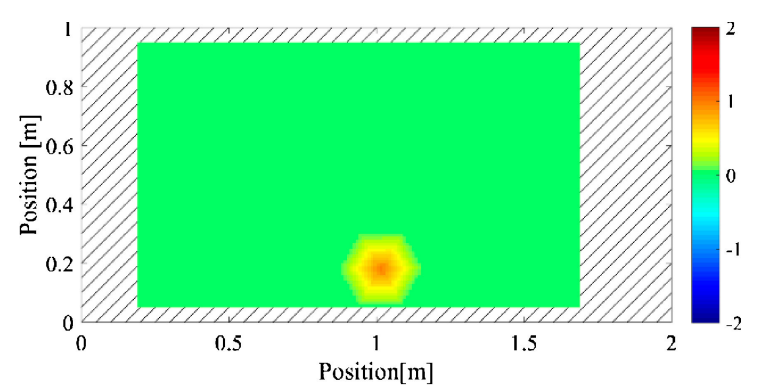

(a) Unit pressure is applied at $(x, y)=(1.0,0.18)$

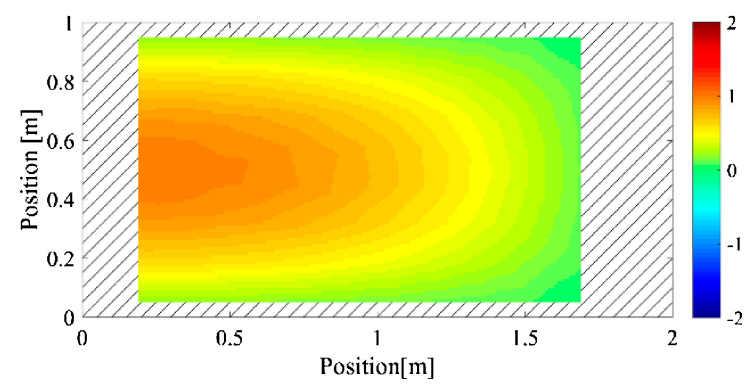

(b) Pressure distribution is applied as in Eq. (5); where, ( $a, b$, $c)=(1.6,0.5,0.5)$

Fig. 4. Examples of the pressure distributions represented by using the pressure discretization map.

at $\left(x_{j}, y_{j}\right)=(1.0,0.18)$ and $p_{i}=0(i \neq j)$. This represents a form of unit pressure mentioned in the following section. Figure 4(b) shows a pressure distribution when the values of $p_{j}$ were given in accordance with the location of the nodes as

$p_{j}\left(x_{j}, y_{j}\right)=\left\{1-\left(\frac{x_{j}-0.19}{a}\right)^{2}\right\}\left\{1-\left(\frac{y_{j}-c}{b}\right)^{2}\right\}$,

where, $(a, b, c)=(1.6,0.5,0.5)$. Through summation of the shape function, the pressure distribution was smoothly represented.

\subsection{Methods: Load identification by inverse analysis approach}

In a linear elastic problem, strain at the $k$ th measurement point, $\varepsilon_{k}(k=1,2, \cdots, m)$, is expressed as

$$
\varepsilon_{k}=\sum_{j=1}^{n} s_{k j} p_{j}, \quad k=1,2, \cdots, m,
$$

where, $s_{k j}$ is a coefficient. The number of strain measurement points was $m=60$ in this study. The coefficient $s_{k j}$ is equivalent to the $k$ th measured strain value when a unit pressure of $p_{j}=1$ and $p_{i}=0(i \neq j)$ is applied. Therefore, the $m$-by- $n$ transfer matrix, $[\mathrm{s}]$, can be calibrated through numerical calculation using the finite element model. In this study, the [s] matrix was calibrated using unit pressuring calculations for $n=85$ times. Once a generalized inverse matrix, $[\mathrm{s}]^{+}$, was calculated, the pressures (loads) of all nodes in the pressure discretization map, namely, the pressure distribution, was determined by 


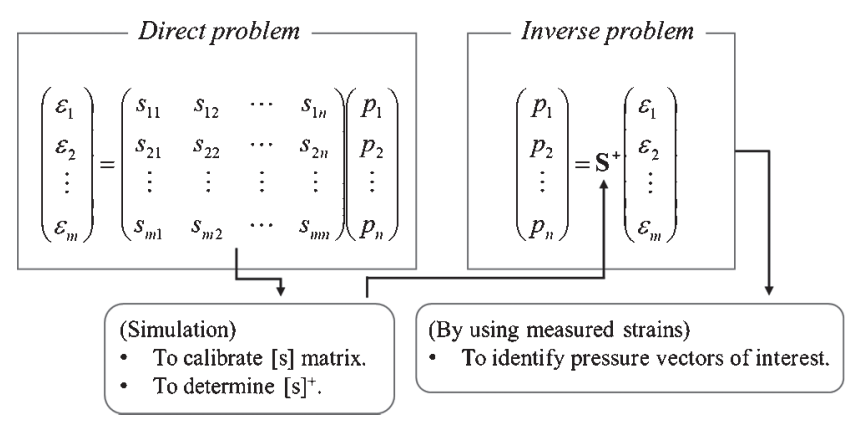

Fig. 5. Schematic diagram of the inverse analysis.

$$
p_{j}=\sum_{k=1}^{m} s_{j k}^{+} \varepsilon_{k}^{m e a s}, \quad j=1,2, \cdots, n
$$

The calculation flow using the pressure vector and strain vector is depicted in Fig. 5. This typical inverse analysis approach tends to be sensitive to the measurement error of strains in nature, when the [s] matrix is ill-conditioned. In order to obtain stable solutions, additional calculation steps, such as truncated singular value decomposition (TSVD), are required.

\subsection{Methods: Load identification by neural network approach}

Instead of using the inverse matrix to represent the strain and load relationship, neural networks can also be applied to solve load identification problems. Using data sets of known strain vectors as inputs and known pressure vectors as outputs, we train the neural network as depicted in Fig. 6. Thereafter, we input unknown strain vectors to the trained neural network to output the unknown pressure vectors of interest.

Although there are many types of neural networks, we consider a simple three-layer, feed-forward neural network. The performance of this type of neural network for load identification has been evaluated in other literature. ${ }^{19,20)}$ The neurons in the input layer output the incoming strain signal directly, which is expressed as

$$
a_{k}^{1}=\varepsilon_{k}, \quad k=1,2, \cdots, m,
$$

where, $a$ represents the output of neurons. The superscript denotes the layer, where " 1 " is input, "2" is hidden and " 3 " is the output layer in this study. The subscript $k$ denotes the $k$ th neuron in the layer. The output of the neurons in the hidden layer is expressed as

$$
a_{i}^{2}=\sigma\left(\sum_{k} w_{i k}^{1} a_{k}^{1}+b_{i}^{1}\right), \quad i=1,2, \cdots, l .
$$

For the hidden layer, the $i$ th neuron forms a weighted sum using the outputs from the previous layer and the weighted coefficient, $w$. The subscript, $i k$, represents the link from the $k$ th neuron in the previous layer to the $i$ th neuron in the current (hidden) layer. The bias, $b$, is added, and the value is activated using the activation function, $\sigma$. In this study, a sigmoid transfer function (hyperbolic tangent) is used. In the same manner, the output of the neurons in the output layer is expressed as

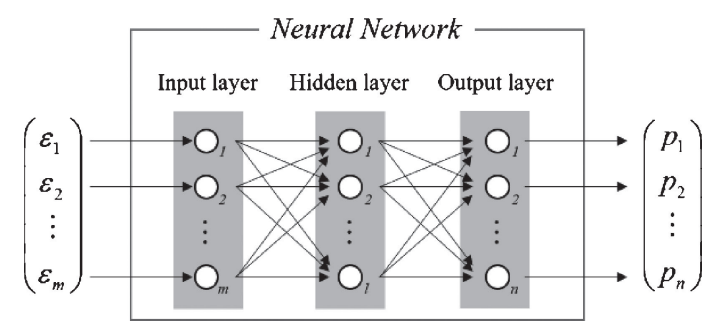

Fig. 6. Schematic diagram of the feed-forward neural network.

$$
a_{j}^{(3)}=\sigma\left(\sum_{i} w_{j i} a_{i}^{(2)}+b_{j}^{(2)}\right), \quad j=1,2, \cdots, n .
$$

The outputs from the output layer directly correspond to the pressure vectors as

$$
p_{j}=a_{j}^{(3)} .
$$

Therefore, the pressure vector to be determined can be expressed using the strain values as

$p_{j}=\left[\sigma\left(\sum_{i} w_{j i}\left[\sigma\left(\sum_{k} w_{i k} \varepsilon_{k}+b_{i}^{(1)}\right)\right]_{i}+b_{j}^{(2)}\right)\right]_{j}$.

Here, comparing Eqs. (7) and (12) illustrates the essential differences between the neural network approach and the inverse analysis approach. In the neural network approach, the strain-to-pressure transfer function, which corresponds to $[\mathrm{s}]^{+}$in the inverse analysis, includes the process of multiplying the weights, adding the biases and non-linear transformation through the activation function. The weights and biases are optimized through iterations in the sense of minimizing the squared errors of the output pressures.

We used the Levenberg-Marquardt back-propagation algorithm for the optimizer. ${ }^{23,24)}$ The initial weights and biases were determined based on the Nguyen-Widrow initialization method. ${ }^{25)}$ The number of neurons in the input and output layers were $m=60$ and $n=85$, respectively, corresponding to the number of strains and pressures, respectively. The number of neurons in the hidden layer is set to be $l=4$ in this study. In this paper, we fix these conditions and do not explore the optimized parameters. Additionally, we rather focus on investigating the essential characteristic of the approach, which is the robustness to errors, as we discuss later.

We prepare training data by numerical simulation using the aforementioned finite element model. A total of 75 pairs of strain and pressure data are applied to train the network. The pressure patterns consist of the parameter combinations of $a=0.8,1.2,1.6,2.0,2.4, b=0.4,0.5,0.6,0.7,0.8$ and $c=0.5,0.7,0.9$ for Eq. (5). To be more precise, five combinations are used for network validation purposes in order to avoid over-fitting, although it may be inevitable to some extent since the 75 pressure patterns are all generated by the same equation, and they are similar to each other. Loss of the validation data is monitored during the optimization iteration. When the loss increases for five iterations, the optimization is stopped and the weights and biases are chosen at the iteration of minimum loss.

In this approach, one can simply train the neural network 
by feeding strain information under known pressures. This approach possesses the potential to eliminate the need of theoretical structure models if training data is available from experiments. One of the drawbacks of the neural network approach is that structural interpretation of the relationship between the input and output is not available in nature. The iterative process must be used to investigate the appropriate selection of training data and learning parameters.

\section{Numerical Simulation Results}

In order to examine the theoretical performance of the load identification, we prepared four example load cases and applied the inverse approach based on transfer matrix and the neural network approach. These four cases were described by individual combinations of $a, b$ and $c$ parameters in Eq. (5). We used theoretical strains that were calculated using the finite element model of the flat panel. In order to evaluate the accuracy of load identification, we introduced a quantitative indicator, called "normalized root mean square error," NRMSE, which is defined as

$$
N R M S E=10 \times \sqrt{\frac{\sum(\hat{p}-p)^{2}}{\sum p^{2}}},
$$

where, $\hat{p}$ is the pressure identified and $p$ is the applied (correct) pressure on each element of the finite element model. Training neural networks includes the process of randomly choosing the initial weights; hence, the neural networks eventually possess random performance in every trial. In order for a fair comparison, when we show NRMSE values for the neural network approach in this section, we show the mean NRMSE value obtained through multiple trials. In detail, we trained neural networks and calculated the load distributions 100 times, and then averaged the NRMSE values of the 100 trials. For figures, we chose and show representative figures of the load identification results that have NRMSE values close to the mean value.

Figure 7 shows four load cases and their identification results. Load cases (i) and (ii) are the ones included in the training data for the neural network approach, while load cases (iii) and (iv) are not included. In case (iii), $a$ and $b$ values are not included in the training parameter, and $b$ is out of the range of training $(0.4 \leq b \leq 0.8)$. In case (iv), $c$ is out of the range of training $(0.5 \leq c \leq 0.9)$. For both approaches, the pressure profiles identified obviously correlate to those applied, which indicates the validity of the methods. The NRMSE values showed higher values, which means lower accuracy, especially for cases (iii) and (iv) of the neural network approach, as shown in Table 1. The load distributions identified are distorted, as shown in Fig. 7. This indicates the nature of the neural network to output better results for the data used to train it. On the other hand, the inverse approach showed relatively equivalent accuracy for the four cases, which indicates high applicability to the arbitral profiles of loads.
Table 1. Values of NRMSE for inverse and neural network approaches. $N R M S E$ of neural network approach is the mean value for 100 trials.

\begin{tabular}{ccc}
\hline Load case & $\begin{array}{c}\text { NRMSE of } \\
\text { inverse approach }\end{array}$ & $\begin{array}{c}\text { NRMSE of } \\
\text { neural network approach }\end{array}$ \\
\hline (i) & 0.65 & 2.5 \\
(ii) & 1.4 & 0.59 \\
(iii) & 0.98 & 4.1 \\
(iv) & 0.85 & 4.1 \\
\hline
\end{tabular}

\section{Reduction of Strain Error Effect}

For both the inverse and neural network approaches, the error in the strain values is critical for identification accuracy. Taking an example of the load distributions as represented in Fig. 7(i), where $(a, b, c)=(1.6,0.6,0.5)$ in Eq. (5), we numerically investigated the effect of the strain error. We simulated 1, 3 and 5\% uniform random strain errors, and examined the identification results. Figure 8 shows the representative results. For the neural network approach, the neural network that output Fig. 7(i) was used. The degradation of load identification was evident for both approaches.

For the inverse approach, we assumed that, in theory, the strain error, $\Delta \varepsilon$, induced the pressure error, $\Delta p$, as

$$
S(p+\Delta p)=\varepsilon+\Delta \varepsilon .
$$

The effect of the relative strain error in the relative pressure error is represented as

$$
\frac{\|\Delta p\|}{\|p\|} \leq \operatorname{Cond}[S] \frac{\|\Delta \varepsilon\|}{\|\varepsilon\|},
$$

where, Cond $[S]$ is the condition number. This reflects it is possible to increase robustness to the strain errors by adjusting the condition numbers. A condition number is defined as a ratio of the maximum and minimum singular values of the [s] matrix as

$$
\operatorname{Cond}[S]=\frac{\lambda_{1}}{\lambda_{m}},
$$

where, $\lambda_{1}$ is the maximum value and $\lambda_{m}$ is the minimum singular value, respectively. It becomes ill-conditioned when the minimum singular value is small compared to the maximum singular value. A typical method to obtain stable solutions is truncated singular value decomposition (TSVD) that truncates small singular values. Setting a threshold, singular values of the $[\mathrm{s}]$ matrix that were smaller than the threshold were set to be zero; hence, the rank of the matrix was reduced. By controlling a threshold, the rank was reduced to an arbitrary range. The rank was changed from 60 (i.e., full rank) to 1 (i.e., minimum rank). For demonstration, $N R M S E$ was calculated to identify the load at each rank. Figure 9 shows the relationship between the NRMSE and rank for the case of $5 \%$ uniform random strain error. The NRMSE decreases as the rank is reduced. Based on NRMSE, this means accuracy is improved; however, excessive smoothing of solutions occurs at the same time. Excessive smoothing results in the loss of distribution profile features. Various methods, such as the L-curve, are applicable for choosing the ap- 
Trans. Japan Soc. Aero. Space Sci., Vol. 62, No. 3, 2019
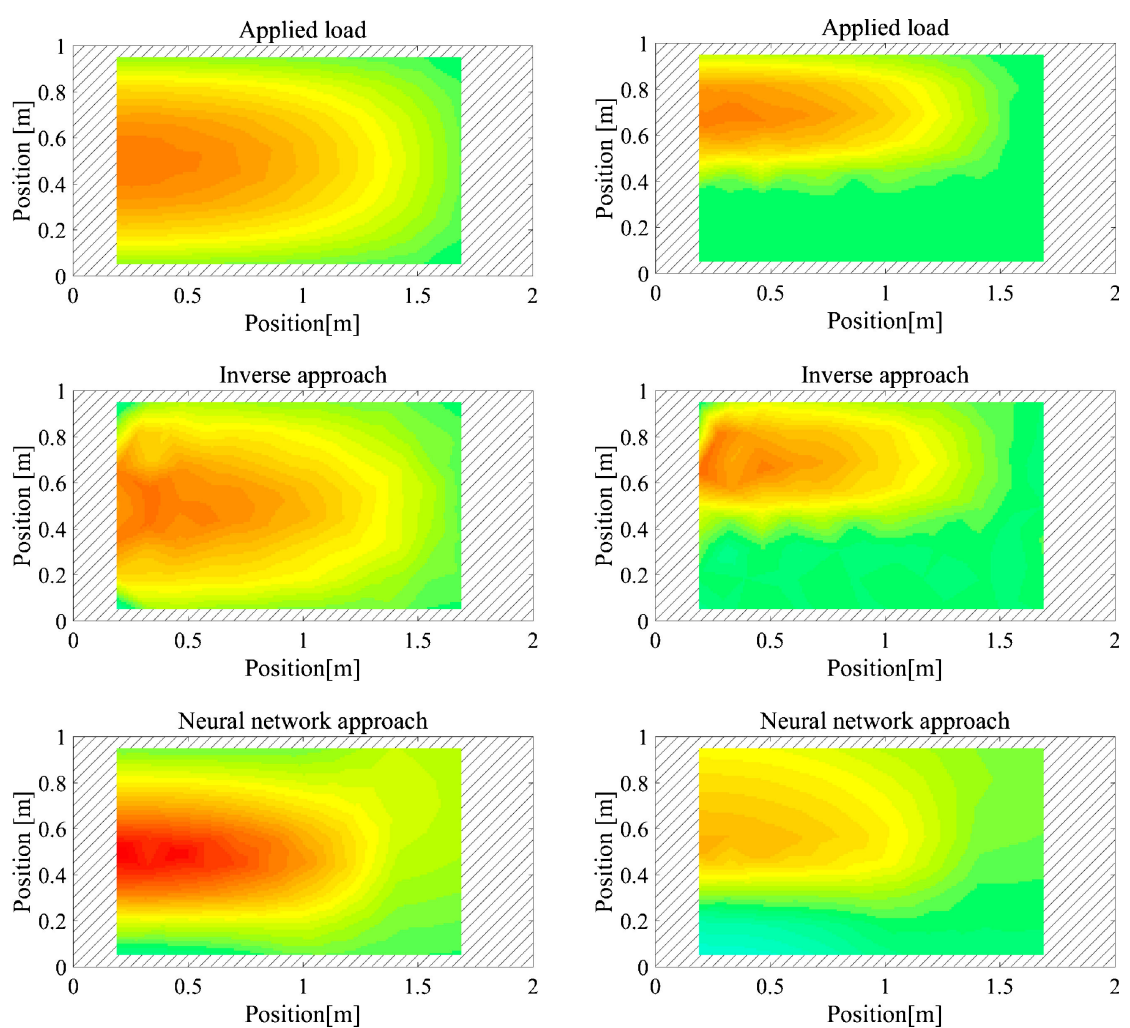

(i) $(a, b, c)=(1.6,0.6,0.5)$

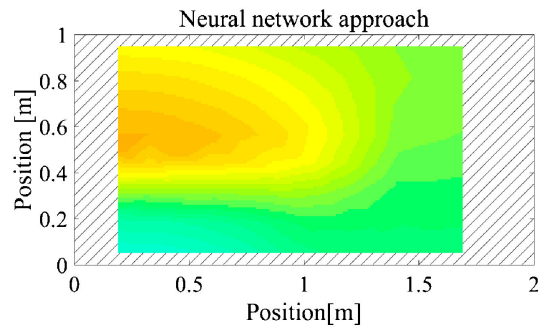

(iii) $(a, b, c)=(1.4,0.3,0.7)$
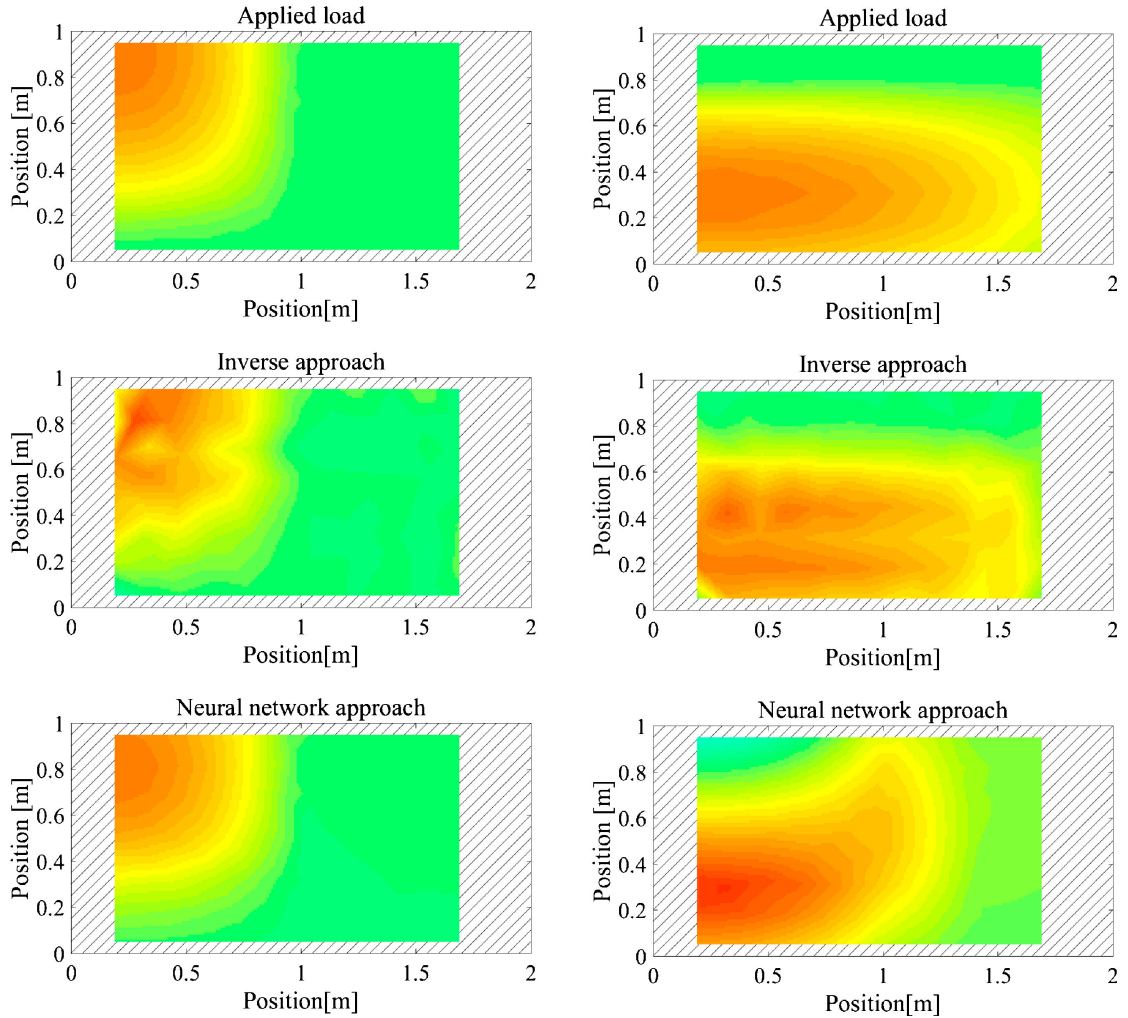

(ii) $(a, b, c)=(0.8,0.8,0.9)$

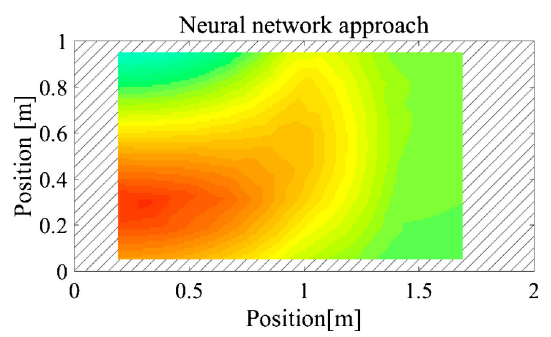

(iv) $(a, b, c)=(2.0,0.5,0.3)$

Fig. 7. Loads to be identified and identification results using the inverse and neural network approaches. Theoretical strains were used. Load distributions as determined utilizing Eq. (5) are represented.

propriate rank. The L-curve method determines the optimal regularization parameter, which is the rank in this case, in the pragmatic sense by obtaining the balance between regularization errors and perturbation errors as described in detail in references. ${ }^{9,26)}$ Using the L-curve method, the optimum rank was determined as 16 . The TSVD method combined with the optimum rank should minimize the effect of strain measurement errors when using inverse analysis. 

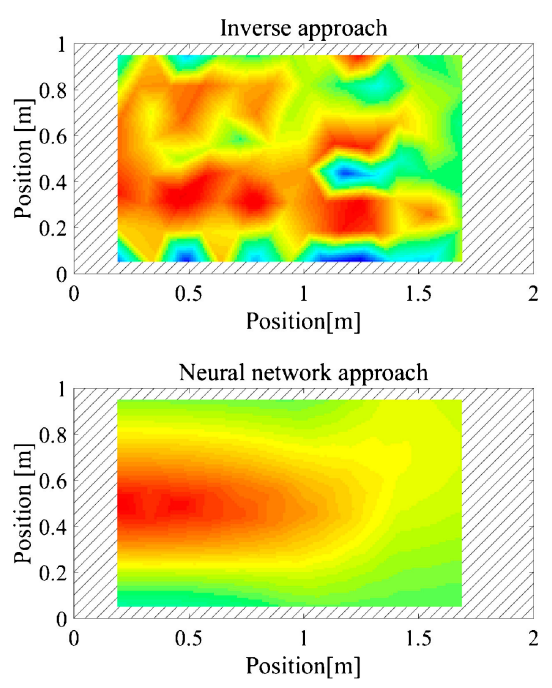

(i) $1 \%$ uniform random strain errors
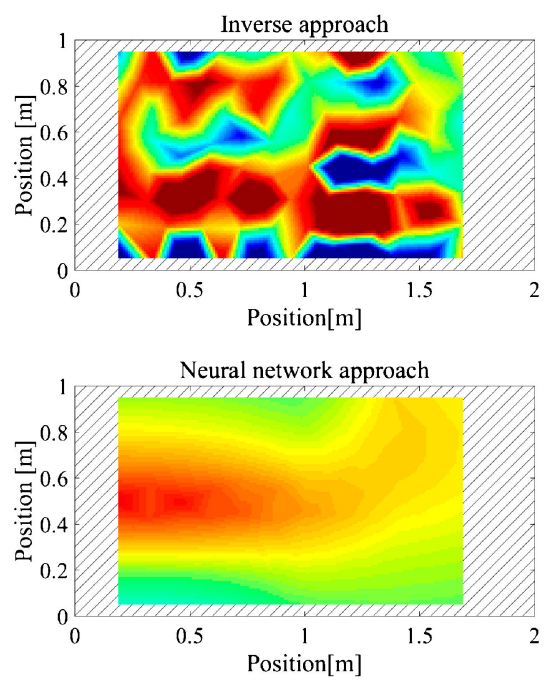

(ii) $3 \%$ uniform random strain errors
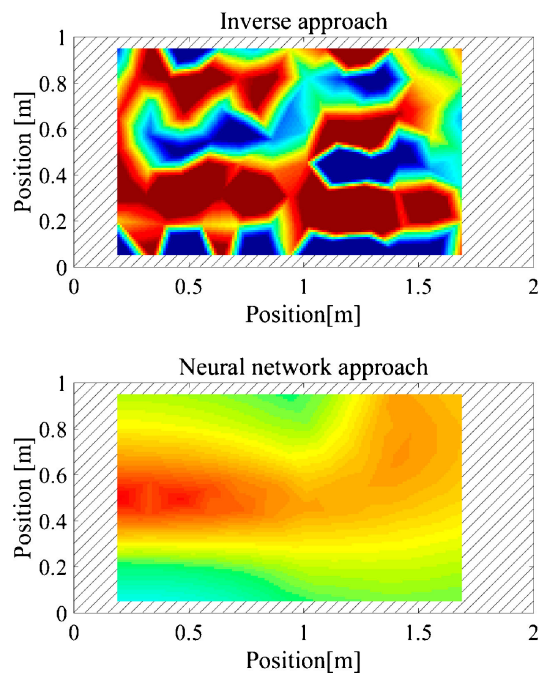

(iii) $5 \%$ uniform random strain errors

Fig. 8. Representative load identification results using the inverse and neural network approaches when strain errors are assumed for theoretical strains.

Target load distribution is the case of $(a, b, c)=(1.6,0.6,0.5)$ in Eq. (5).

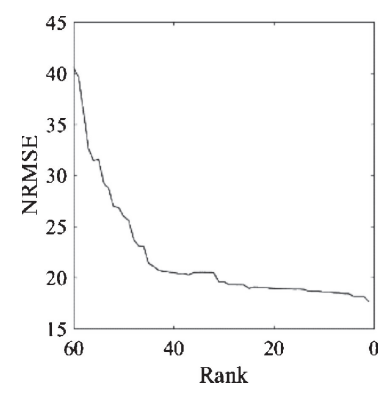

Fig. 9. Relationship between $N R M S E$ and the rank of the inverse matrix $[\mathrm{s}]^{+}$for the case of $(a, b, c)=(1.6,0.6,0.5)$ in Eq. (5) when $5 \%$ uniform random strain errors are assumed.

For the neural network approach, it is difficult to directly adjust the weights and biases in Eq. (12). The weights and biases should be further optimized through training so that they output robust results against the strain error. One way to improve robustness is to add training data that includes artificial strain errors. We prepared another 100 data for the case of Fig. 7(i), to which we added 5\% uniform random strain errors. We updated the neural network using 175 training data in total.

Figure 10 shows the representative load identification results using the inverse approach with the TSVD method and the neural network approach using the updated neural network. Compared to the results without the error reduction methods, as shown in Fig. 8, the improvement in accuracy is evident. For the inverse approach using TSVD method, the load distribution was relatively distinguishable when the strain error was $1 \%$; however, the elliptical profile was unclear. More than $1 \%$ strain error caused critical distortion of the results. On the other hand, the updated neural network approach showed high robustness to the strain errors, up to $5 \%$. The load distribution profiles did not suffer the error effect and output constant results for $1-5 \%$ error cases.

In order to examine the error effect quantitatively, we applied the Monte Carlo method. We calculated the load identification with strain errors for 1,000 rounds, and then calculated the mean value for NRMSE. Figure 11 shows the mean NRMSE for the inverse and neural network approaches with and without the error reduction methods. The NRMSE values revealed linearity with the strain error amplitude for all of the approaches. The TSVD method largely contributed to suppressing the error in a relative sense; however, the distorted results in Fig. 10 indicate the inevitable necessity of a certain assumption, such as a distribution function for aerodynamic loads to obtain stable solutions. The updated neural network approach showed the smallest absolute NRMSE values and the sensitivity to the strain error among the four approaches, which resulted in successful identification of the strain error up to $5 \%$. This indicates the potential of the neural network approach identifying arbitrary distributions of loads. The training data influences identification performance. It is believed that it will be easy to add artificial strain errors to the training data. By training the neural network with possible load distributions 

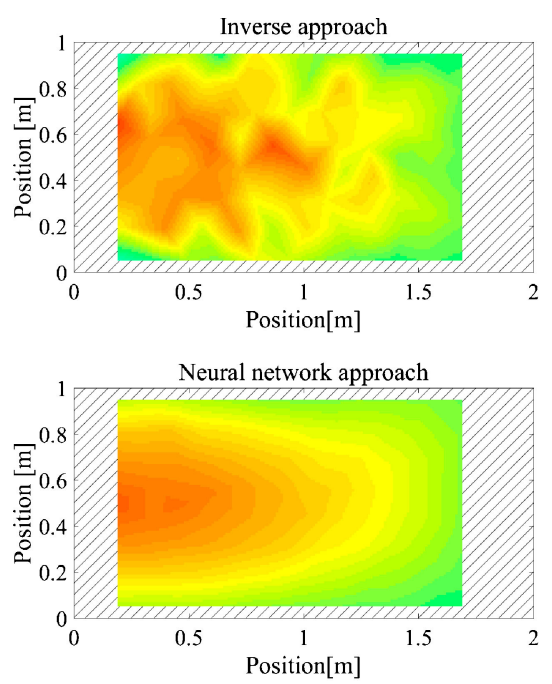

(i) $1 \%$ uniform random strain errors
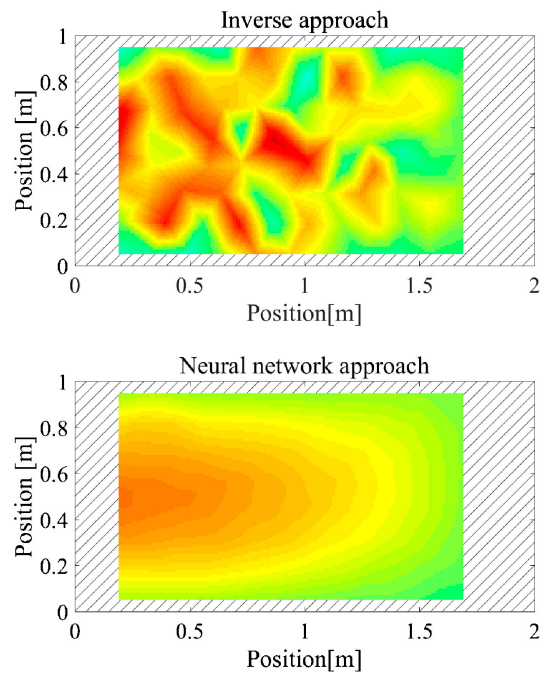

(ii) $3 \%$ uniform random strain errors
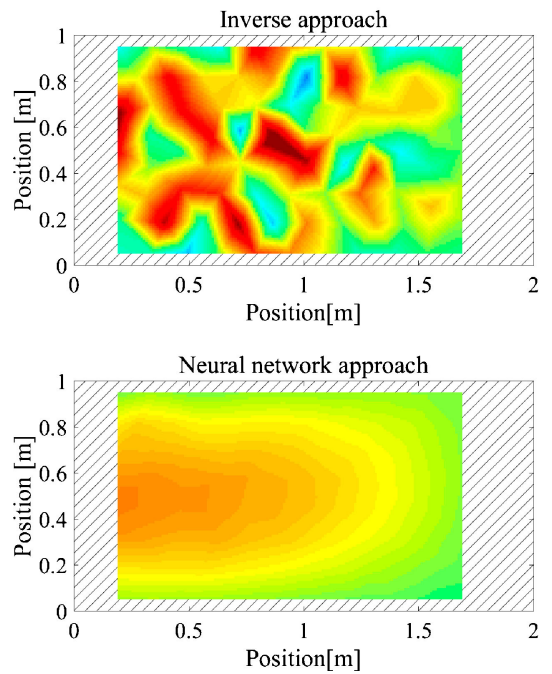

(iii) $5 \%$ uniform random strain errors

Fig. 10. Representative load identification results using the inverse approach with the TSVD method and the neural network approach using an updated neural network when strain errors are assumed for the theoretical strains.

Target load distribution is the case of $(a, b, c)=(1.6,0.6,0.5)$ in Eq. (5).

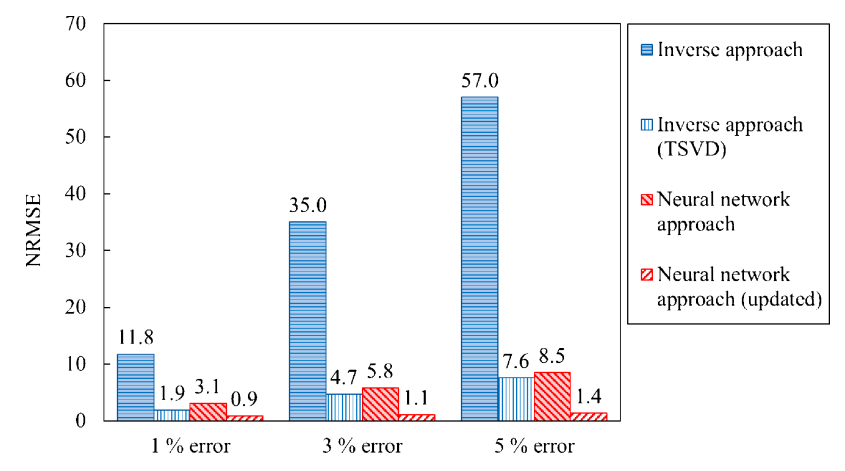

Fig. 11. Relationship between NRMSE and the strain errors for the inverse and neural network approaches applying individual error reduction methods.

NRMSE is the mean value for 1,000 rounds of calculations using random errors.

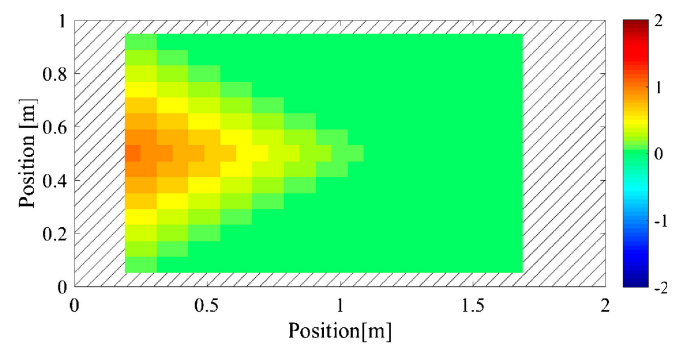

(a) Applied load

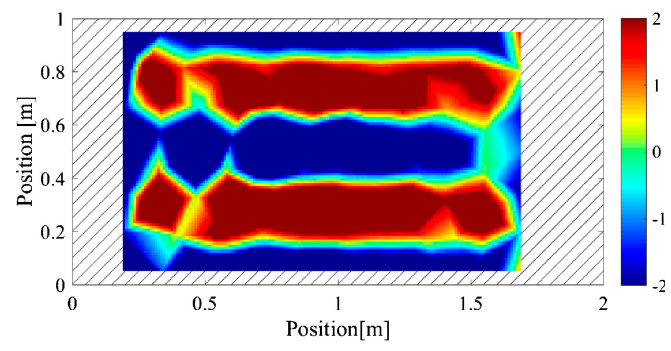

(b) Inverse approach

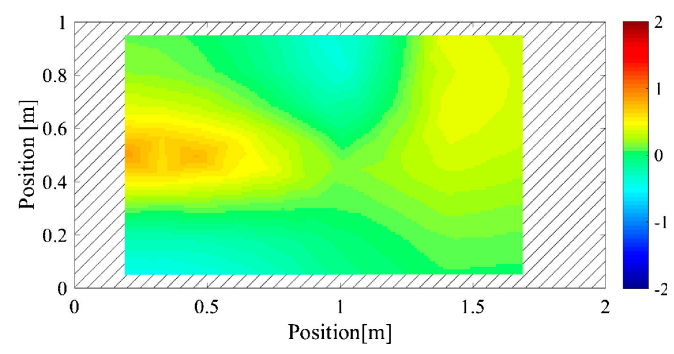

(c) Neural network approach

Fig. 12. Experimental loads applied to the flat panel and identification results.

Pressure values were normalized by the maximum pressure of $460 \mathrm{MPa}$.

and expected strain errors, this approach would be a powerful option for load identification.

\section{Experimental Results}

For experimental investigation, we set aluminum square plates on a flat panel as, shown in Fig. 2, and applied pres- 


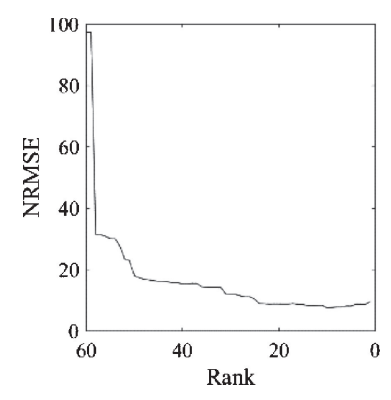

Fig. 13. Experimental relationship between NRMSE and rank of the inverse matrix $[\mathrm{s}]^{+}$

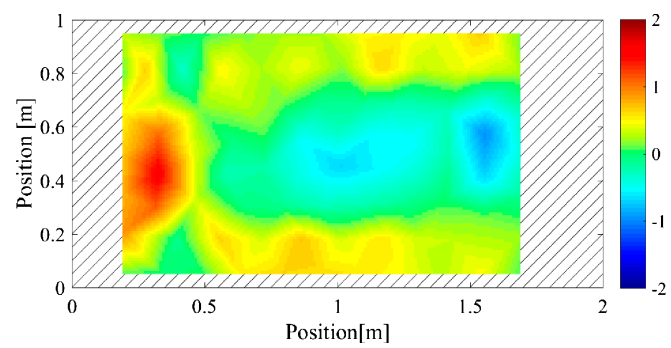

Fig. 14. Load identification results for the inverse approach when the optimum rank $=34$.

sure distributed over the area, as represented in Fig. 12(a). The pressure values were normalized by the maximum pressure of $460 \mathrm{MPa}$. Using 60 strain gauges, we conducted load identification based on inverse and neural network approaches. For the neural network approach, the neural network that resulted in the output shown in Fig. 7(i) was used. The results are shown in Fig. 12(b) and (c). The values of NRMSE were 102 for the inverse approach and 8.6 for the neural network approach. Load identification was obviously unsuccessful. As discussed in a previous section, the main reason is due to the inherent error of the strain gauges. Additionally, it is believed that all of the training data applied in the neural network approach was an elliptical form of pressure, while the experimental load was a linear profile. The identification results shown in Fig. 12(c) have a rather elliptical form, and this is because the neural network fits it well.

In order to reduce the strain error effect for the inverse approach, we applied the TSVD method. Figure 13 shows the relationship between the NRMSE and rank. The NRMSE decreases as the rank is truncated, and then increases gradually after a certain point. This gradual increase reflects the excessive smoothing of solutions. In this experiment, the L-curve method determined the optimum rank as 34. Figure 14 shows the load identification result for the inverse approach with the TSVD method when the rank is 34 . The NRMSE value was 14.1. The NRMSE was reduced; hence, there was a relative improvement in accuracy compared to the full rank. However, once again, it was hard to distinguish the loads applied from the identification results, as shown in the simulation results.

For the neural network approach, we added one load data for training that reflected the load applied. We prepared a load case as shown in Fig. 15, which represents the experi-

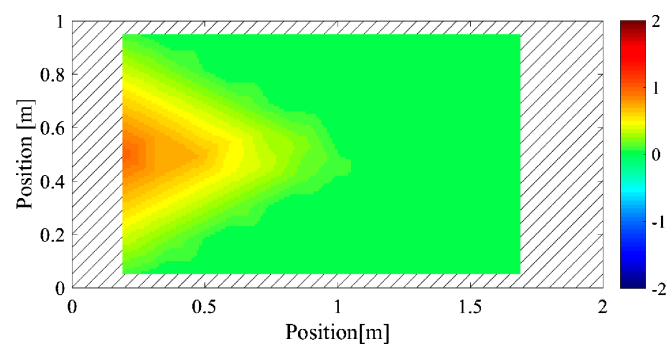

Fig. 15. Newly added training data reflecting the experimental load.

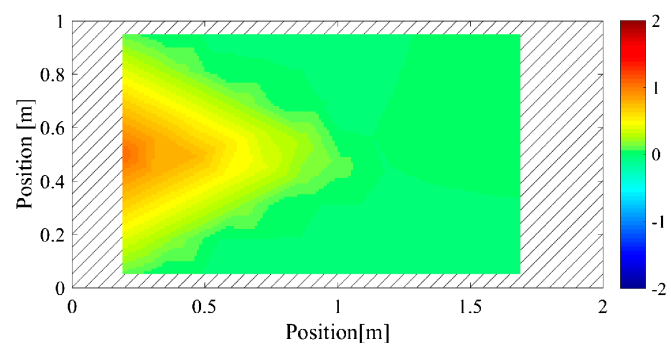

Fig. 16. Load identification results using the updated neural network. A total of 176 data was used to train the neural network.

mental load using the pressure discretization map. In addition, in order to include the error reduction, we added 100 training data that included artificial strain errors. We prepared the 100 data for the case of Fig. 15, to which we added $5 \%$ uniform random strain errors. In total, we added 101 training data to the original 75 data, and we updated the neural network using them. Figure 16 shows the load identification results. The NRMSE value was 1.3 , and identification was successful. It was experimentally demonstrated that the training including strain errors enhances identification robustness. Another positive effect was also observed. Figure 17 shows the load identification results using the updated neural network for the load cases in Fig. 7, and Table 2 shows the NRMSE values. It is noteworthy that we used and updated the respective neural networks that output the results in Fig. 7; that is, we used and updated four neural networks corresponding cases (i)-(iv). Table 2 shows the $N R M S E$ of the respective neural networks, whereas Table 1 shows the mean NRMSE value for 100 neural networks per case. Therefore, there is a slight variation in the NRMSE value for the original neural networks. In load case (ii), the identification accuracy was slightly degraded due to the update, but it was originally very high and sufficient to identify the distribution profile. In all of the other cases, accuracy improved. This indicates that increasing the amount of training data, as well as the variety, contributed to reducing over-fitting of the network to the training data consisting of elliptical load patterns, and consequently resulting in better accuracy.

\section{Conclusions}

We proposed and investigated two approaches, an inverse approach and a neural network approach, to identify load distributions on a flat panel using strain measurement values. We used a pressure discretization map for both approaches, 


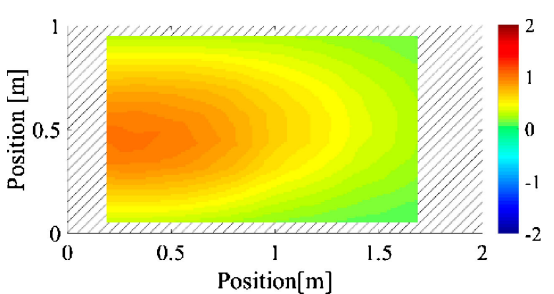

(i) $(a, b, c)=(1.6,0.6,0.5)$

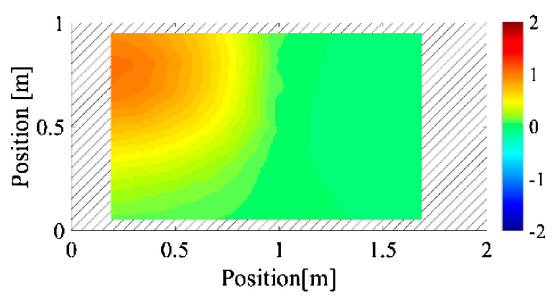

(ii) $(a, b, c)=(0.8,0.8,0.9)$

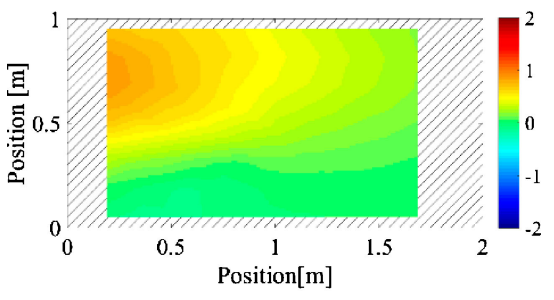

(iii) $(a, b, c)=(1.4,0.3,0.7)$

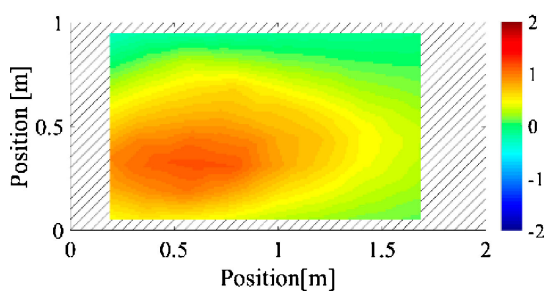

(iv) $(a, b, c)=(2.0,0.5,0.3)$

Fig. 17. Load identification results for simulated loading cases using the updated neural network.

A total of 176 data was used to train the neural network.

Table 2. Values of NRMSE for the original and updated neural network approaches.

\begin{tabular}{ccc}
\hline Load case & $\begin{array}{c}\text { NRMSE of } \\
\text { original neural network }\end{array}$ & $\begin{array}{c}\text { NRMSE of } \\
\text { updated neural network }\end{array}$ \\
\hline (i) & 2.5 & 0.92 \\
(ii) & 0.59 & 0.75 \\
(iii) & 4.1 & 3.1 \\
(iv) & 3.9 & 2.3 \\
\hline
\end{tabular}

thereby enabling load identification with arbitrary distribution profiles.

Numerical simulation results verified both approaches. Although the neural network output better results for the data used to train it, it was also applicable to the load cases that were not included in the training data. The inverse approach showed relatively equivalent accuracy to different load cases, which indicates high applicability to arbitral profiles of loads where strain error does not exist.
When strain measurement error exists, the inverse approach with the TSVD method for the error reduction is not capable of successfully identifying load distributions when more than $1 \%$ artificial strain error is applied during simulation. This was the result for the experiment as well. This inevitably indicates the necessity for certain assumptions, such as a distribution function for aerodynamic load, in order to obtain stable solutions using the inverse approach. On the other hand, the updated neural network approach, which trains neural networks with the data including artificial strain errors, showed high robustness to the strain errors for both simulation and experiment. In the simulation results, the NRMSE for the case with $5 \%$ strain error was only 1.4 , whereas the NRMSE for the case with $1 \%$ strain error in the inverse approach with the TSVD method was 1.9. Training in the case of using artificial strain errors for the experimental load contributed to better accuracy, even for other load cases. These results indicate the potential of the neural network approach to identify the arbitrary distribution of loads.

The training data influences the identification performance of the neural network approach. By training the neural network with the possible load distributions and expected strain errors, this approach becomes a powerful option for load identification.

\section{Acknowledgments}

This work was supported by JSPS KAKENHI Grant Number JP17K14878.

\section{References}

1) Aktepe, B. and Molent, L.: Management of Airframe Fatigue through Individual Aircraft Loads Monitoring Programs, International Aerospace Congress, Adelaide, Australia, 1999, pp. 1-14.

2) Molent, L. and Aktepe, B.: Review of Fatigue Monitoring of Agile Military Aircraft, Fatigue Fract. Eng. Mater. Struct., 23 (2000), pp. 767-785.

3) Farrar, C. R. and Worden, K.: An Introduction to Structural Health Monitoring, Phil. Trans. R. Soc. A, 365 (2007), pp. 303-315.

4) Molent, L. and Inan, S.: Specifications for an Unified Strain and Flight Parameter Based Aircraft Fatigue Usage Monitoring System, DSTO International Conference on Health and Usage Monitoring, Melbourne, Australia, 2001, pp. 53-67.

5) Farrar, C. R. and Lieven, N. A. J.: Damage Prognosis: the Future of Structural Health Monitoring, Phil. Trans. R. Soc. A, 365 (2007), pp. 623-632.

6) Prosser, W. H., Allison, S. G., Woodard, S. E., Wincheski, R. A., Cooper, E. G., Price, D. C., Hedley, M., Prokopenko, M., Scott, D. A., Tessler, A., and Spangler, J. L.: Structural Health Management for Future Aerospace Vehicles, 2nd Australian Workshop on Structural Health Monitoring, Melbourne, Australia, 2004.

7) Tikhonov, A. N. and Arsenin, V. Y.: Solution of Ill-Posed Problems, Winston, New York, 1977.

8) Tarantola, A.: Inverse Problem Theory and Methods for Model Parameter Estimation, SIAM, Philadelphia, Pennsylvania, 2005.

9) Hansen, P. C.: Discrete Inverse Problems: Insight and Algorithms, SIAM, Philadelphia, Pennsylvania, 2010.

10) Aster, R. C., Borchers, B., and Thurber, C. F.: Parameter Estimation and Inverse Problems, 2nd ed., Academic Press, Cambridge, Massachusetts, 2012.

11) Schnur, D. S. and Zabaras, N.: Finite Element Solution of Two-dimensional Inverse Elastic Problems Using Spatial Smoothing, Int. J. 
Numer. Meth. Eng., 30 (1990), pp. 57-75.

12) Maniatty, A., Zabaras, N., and Stelson, K.: Finite Element Analysis of Some Inverse Elasticity Problems, J. Eng. Mech., 115 (1989), pp. 1303-1317.

13) Maniatty, A. and Zabaras, N.: Method for Solving Inverse Elastoviscoplastic Problems, J. Eng. Mech., 115 (1989), pp. 2216-2231.

14) Maniatty, A. M. and Zabaras, N. J.: Investigation of Regularization Parameters and Error Estimating in Inverse Elasticity Problems, Int. J. Numer. Meth. Eng., 37 (1994), pp. 1039-1052.

15) Martin, T. J., Halderman, J. D., and Dulikravich, G. S.: An Inverse Method for Finding Unknown Surface Tractions and Deformations in Elastostatics, Comput. Struct., 56 (1995), pp. 825-835.

16) Shkarayev, S., Krashantisa, R., and Tessler, A.: An Inverse Interpolation Method Utilizing In-flight Strain Measurements for Determining Loads and Structural Response of Aerospace Vehicles, 3rd International Workshop on Structural Health Monitoring, Stanford, California, USA, 2001.

17) Coates, C. W. and Thamburaj, P.: Inverse Method Using Finite Strain Measurements to Determine Flight Load Distribution Functions, $J$. Aircraft, 45 (2008), pp. 366-370.

18) Nakamura, T., Igawa, H., and Kanda, A.: Inverse Identification of Continuously Distributed Loads Using Strain Data, Aerosp. Sci. Technol., 23 (2012), pp. 75-84.

19) Cao, X., Sugiyama, Y., and Mitsui, Y.: Application of Artificial Neural Networks to Load Identification, Comput. Struct., 69 (1998), pp. 63-78.

20) Trivailo, P. M. and Carn, C. L.: The Inverse Determination of Aerodynamic Loading from Structural Response Data Using Neural Networks, Inverse Prob. Sci. En., 14 (2006), pp. 379-395.

21) Wada, D. and Sugimoto, Y.: Inverse Analysis of Aerodynamic Loads from Strain Information Using Structural Models and Neural Networks, Proc. SPIE, 10168 (2017), 101680W.

22) Wada, D., Igawa, H., and Kasai, T.: Vibration Monitoring of a Helicopter Blade Model Using the Optical Fiber Distributed Strain Sensing Technique, Appl. Optics, 55 (2016), pp. 6953-6959.

23) Levenberg, K.: A Method for the Solution of Certain Problems in Least Squares, Quart. Appl. Math., 2 (1944), pp. 164-168.

24) Marquardt, D.: An Algorithm for Least-Squares Estimation of Nonlinear Parameters, SIAM J. Appl. Math., 11 (1963), pp. 431-441.

25) Nguyen, D. and Widrow, B.: Improving the Learning Speed of 2-Layer Neural Networks by Choosing Initial Values of the Adaptive Weights, Proceedings of the International Joint Conference on Neural Networks, Vol.3, 1990, pp. 21-26.

26) Hansen, P. C. and O'Leary, D. P.: The Use of the 1-Curve in the Regularization of Discrete Ill-posed Problems, SIAM J. Sci. Comput., 14 (1993), pp. 1487-1503.

Yasuyuki Miyazaki Associate Editor 